little blood-clot but none was found in the pelves or ureters. The bladder was distended with clear, yellow urine, which gave no hæmoglobin reaction when tested with tincture of guaiacum and ozonic ether.

I am indebted to Dr. J. E. Beggs, medical superintendent, for permission to publish this case.

Tooting, S.W.

\section{SOME ADDITIONAL REMARKS ON THE ETIOLOGY AND DIAGNOSIS OF ACUTE DILATATION OF THE STOMACH.}

By H. CAMPBELL THOMSON, M.D., F.R.C.P. LoND., assistant PHYSICIAN TO THE MIDDLESEX HOSPITAL.

IN my recently published book on the subject of acute dilatation of the stomach $\mathrm{I}$ have discussed at some length the different theories which have been put forward to account for the disease, and while in no way denying that some cases may be obstructive in origin I inclined to the belief that a primary paralysis of the organ must be regarded as the underlying cause in the majority of the cases. Those who support the theory that obstruction is the main cause consider that under certain conditions within the abdomen the superior mesenteric artery may exercise a constricting force upon the duodenum, and the conditions necessary thus to produce this constriction are thought to be fulfilled by the displacement of collapsed intestine into the pelvis by which such a tension is put upon the mesentery that the cord-like superior mesenteric artery becomes converted into a constricting band and obliterates the lumen of the duodenum. There are many arguments against this theory which I have discussed elsewhere and need not again refer to here, but the main objection to the adoption of this view lies in the fact that the point at which distended bowel passes into collapsed bowel varies in different cases coils of intestine is shown in the accompanying figure which is reproduced trom a photograph taken at the time of the necropsy, and it is obvious that in this case the superior mesenteric artery cannot be considered as the constricting force nor were there any signs of any other constricting force having been present.

In the absence of any constriction a primary paralytic. condition would seem to give an adequate explanation of this case, as it does in so many others, and, as I have before pointed out, there is no obvious reason why, if a primary paralysis is assumed to take place in the stomach, we should suppose that paralysis to be necessarily limited to the stomach. The nervous connexions between the vagi and abdominal plexuses are very complex, and it seems reasonable to suppose that the paralysis which involves the stomach may in different cases extend variable distances along the intestine and such variable extension would adequately explain the fact that the distended bowel terminates at different levels. Reference in my former writings will also be found to the probable relationship between acute dilatation of the stomach and the paralytic distension of the intestines which occurs during cases of acute peritonitis. It is, of course, common, and indeed usual, to find the intestines distended in cases of peritonitis and there can be little doubt that the distension is due to paralysis of the gut walls consequent to the intlammation of the peritoneum. While in these cases distension of the intestine is usually the predominating feature, both during life and after death, the necropsy often shows that the stomach has also to some extent participated in the condition and occasionally the gastric symptoms form the most prominent feature of the case and may for the time even mask all the symptoms of the underlying peritonitis. This fact assumes considerable importance when it is remembered that peritonitis is the condition which clinically most closely resembles acute dilatation of the stomach and for which many primary cases of the latter have been mistaken. We have thus in every case in which symptoms of acute dilatation of the stomach occur after an abdominal operation, or under other conditions in which aperitonitis would

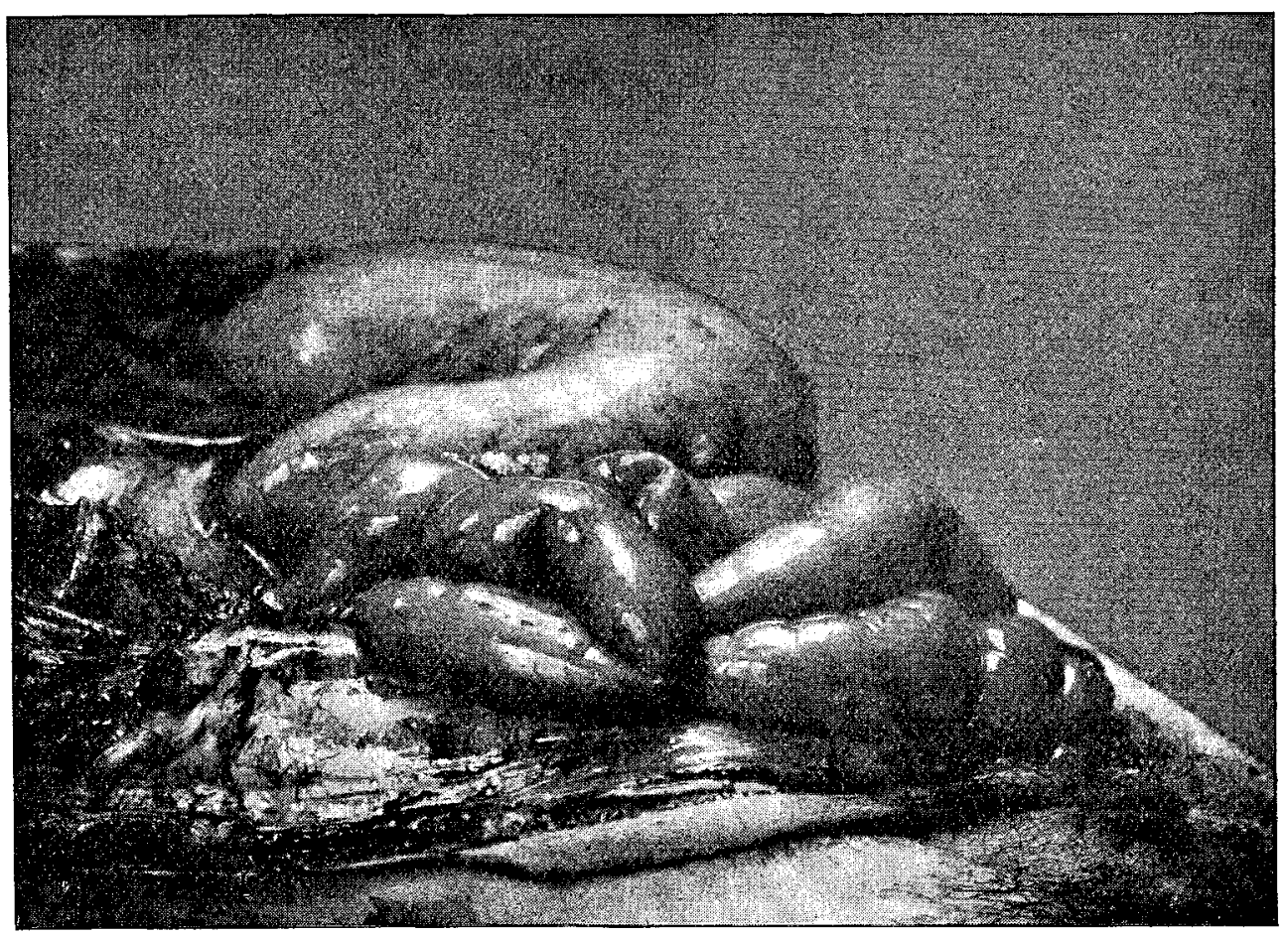

Acute dilatation of the stomach and upper eight feet of intestine.

which it obviously would not do if the situation of the constricting force were a constant one as it would be in the case of the superior mesenteric artery. I quoted cases in my book showing that sometimes only the first part of the duodenum shared in the dilatation while in others the distension extended some inches along the jejunum. More recently I have had an opportunity of making a necropsy upon a case in which acute dilatation of the stomach was accompanied by distension of eight feet of small intestine, the distension passing abruptly into collapsed bowel at that point. The appearance of the distended stomach and upper be likely to arise, to decide whether the dilatation is primary condition or whether it is only the predominating feature of a general peritonitis. A case which I recently had an opportunity of seeing with Dr. Comyns Berkeley well illustrates this condition and also the difficulty which one may have in arriving at a correct diagnosis when there is a possibility of peritonitis co-existing, although there may be no obvious reasons which would leadrone to suspect its presence.

The patient was a female, aged 32 years, and had previously had five children. Sl.e had passed uneventfully 
through a sixth pregnancy and on the evening of Jan. 5th, 1902, intermittent pains came on and it was considered that labour had commenced. On the evening of the 7th the pain became continuous and more distressing and about the same time the patient commenced to vomit some yellow fluid. The pain and vomiting continued and were for some hours accompanied by alarming symptoms of collapse, but the child was safely delivered and after that the symptoms subsided; the pulse fell from 130 to 80 and the temperature was normal. This respite was, however, very brief and six hours later the pulse had risen to 136 , the respirations were 60 , and there was continuous vomiting and great collapse but no pain. It was at this juncture that Dr. Berkeley first saw the patient and on examination he found signs indicating great dilatation of the stomach. The organ appeared to reach within two inches of the pubes. The liver dulness was partially obliterated and the lower lobe of the left lung was pushed up, so that the percussion note over the lower part of the left side of the chest was hyper-resonant. The diagnosis was further confirmed by the elicitation of a marked succussion splash and by the presence of frequent vomiting of large quantities of yellowish fluid without any effort. Dr. Berkeley kindly asked me to see the case and we came to the conclusion that the predominating symptoms were those of acute dilatation of the stomach, and from the apparently normal confinement which had taken place only a few hours before and in the absence of abdominal pain and tenderness there did not seem any justification for supposing there was any underlying cause other than shock. We therefore, with a view of alleviating the more distressing symptoms, passed a soft tube into the stomach and drew off over three pints of fluid, after which the patient for a time expressed herself as considerably relieved but she eventually died about six hours later. A post-mortem examination showed the presence of general purulent peritonitis which had arisen from the rupture of an ovarian abscess. The stomach was moderately distended and had evidently undergone some contraction after the evacuation of the fluid, as has frequently been noticed in other cases.

In this instance, then, the acute dilatation of the stomach was secondary to the peritonitis and therefore merely a symptom of a much graver condition. It is, of course, most important from the point of view of both prognosis and treatment to distinguish, if possible, between such a case and one in which the stomach factor is either the primary sondition or the threatening fatal complication of some less serious malady.

Queen Anne-street, W.

\section{NOTES ON THE THERAPEUTIC USES OF THE SALSOMAGGIORE WATERS.1}

\section{Bx G. SANDISON BROCK, M.D. EDIN. \& RoME, F R.S. EDIN.}

IN the admirable report of THE LANCET Special Analytical and Sanitary Commission on the Iodo-bromo-saline Waters of Salsomaggiore ${ }^{2}$ reference is made to my own observations bearing upon the therapentic uses of the waters. At the risk of repetition I shall bere endeavour to arrange these as far as possible in the order of their importance and at the same time to supplement to some extent what has already appeared in THE LANCE'T'S report.

The uses to which the waters of Salsomaggiore are now put, as well as the methods of employing them, have been of the first few cases recorded by Berzieri, the village practitioner of Salsomaggiore, to whom the original inspiration came (in 1839) of utilising them for therapeutic purposes. The first case in which he used the baths was one of caries of the bones of the foot, no doubt tuberculous, and to this day the waters continue to enjoy the reputation earned by the success of that treatment in a great variety of tuberculous lesions, whether in bones, joints, glands, or skin. It is, perhaps, chiefly for this class of cases that the waters are most esteemed amongst the common people in Italy, the various communes sending for treatment to the sanatorium of Salsomaggiore batches of patients, mostly of this kind, every three

1 For much information regarding the therapeutics of the waters I am indebted to my colleague at the baths, Professor Zoja. weeks throughout the summer and paying their expenses. No better testimonial to the efficacy of the waters could be given than the fact that the same communes, encouraged by the great benefit derived, continue to send such patients year after year, and that in many instances these patients return from time to time of their own accord and at their own expense when assailed by recurrences of their maladies. No doubt the change of air and the better food which such patients obtain at Salsomaggiore contribute largely to the success of the treatment in these cases; nevertheless, they must still be regarded as amongst the most promising of those indicated for the Salsomaggiore cure.

The alterative, absorbent, and healing properties displayed by the waters in these scrofulous cases naturally led to an extension of their use to all affections where in connexion with a localised morbid process there existed open sores, sinuses, inflammatory swellings, or exudations. Their beneficent action in the chronic stages of inflammatory diseases forms, indeed, the basis of their fame at the present day. In the acute stages they are generally regarded as contra-indicated (although this is by no means a settled question), but the acute stage once over all are agreed that great benefit is derived from their use in such affections. In cases of appendicitis where a certain amount of pain and swelling remain after an acute attack or where an abscess has been opened or has burst leaving painful adhesions or exudations behind in the neighbourhood of the colon the absorbent and healing effects of the baths are often very remarkable. For sequelæ of peritonitis, of pleurisy, and of synovitis, for arthritic troubles after dislocations, for the thickening of bone and periosteum following fractures and other injuries, or indeed in most chronic inflammatory processes accompanied by pain, stiffness, swelling, or deformity, the soothing or stimulating properties of these waters, or of applications of the mud deposited from them, are found to be highly beneficial. Especially satisfactory are the results obtained in arthritis consequent upon acute rheumatism or relapses of that malady, as well as in that occasioned by gout. In these cases the baths exercise a strongly soothing and absorbent influence locally, and at the same time stimulate powerfully the excretory functions of the body, enabling the system to throw off rapidly the poisonous waste products by the skin, the lungs, and the kidneys.

The remarkable absorbent effects of the waters have led to their very extensive employment for diseases of women. It is, perhaps, in this connexion that they are most known and made use of in Italy amongst the well-to-do classes. All possible varieties of gynæcological ailments find their way to Salsomaggiore, and it is really wonderful how many different maladies in that large category of diseases find relief from the treatment. This is explained by the fact that the greater number are of an inflammatory nature, in the chronic stage so favourable to the healing action of the waters. Amongst those most amenable to the treatment are the various inflammatory affections of the uterus and adnexa-to wit, cervicitis, endo-, para-, and peri-metritis, salpingitis, pelvic peritonitis, and pelvic cellulitis. Lacerations, displacements, and new growths may derive benefit as far as certain secondary effects produced by them are concerned. Sterility is often cured, as might be expected when the cause producing it is susceptible of removal, by a course of the baths. Functional derangements of the sexual organs of the most diverse kind often yield to the treatment - such us amenorrhœea, menorrhagia, dysmenorrhœea, or irregularity of menstruation-a result due probably to a general tonic effect which the waters exercise upon the nutrition of the body.

The large quantity of iodine present in the waters has suggested their use in diseases due to infection by specific germs other than that of tubercle, and they are, in fact, found of great value in the later stages of syphilis (both for the secondary manifestations in the skin, the mucous membrane, and the periosteum, and for the tertiary changes in the bones and the organs), as well as for the sequelæe of gonococcic infection, whether in the genital organs themselves (as urethritis, orchitis, metritis, \&c.) or in other parts of the body (post-gonorrhoeal rheumatism, arthritis, and synovitis).

A considerable number of neurasthenic and anæmic patients also benefit, often quite remarkably, by the Salsomaggiore treatment. In those cases the restfulness and quiet of the place itself must greatly contribute to the successful result, 